\section{OPEN ACCESS}

Edited by:

Martin Reuter,

University of Bonn, Germany

Reviewed by:

Emiliano Ricciardi,

IMT School for Advanced Studies

Lucca, Italy

Kirsten Hilger,

Julius Maximilian University of Würzburg, Germany

*Correspondence:

Fabio Sambataro

fabio.sambataro@unipd.it

Specialty section:

This article was submitted to

Personality and Social Psychology, a section of the journal

Frontiers in Psychology

Received: 15 September 2020

Accepted: 05 February 2021

Published: 04 March 2021

Citation:

Olivo D, Di Ciano A, Mauro J,

Giudetti L, Pampallona A, Kubera KM,

Hirjak $D$, Wolf $R C$ and Sambataro F

(2021) Neural Responses of

Benefiting From the Prosocial

Exchange: The Effect of Helping

Behavior. Front. Psychol. 12:606858.

doi: 10.3389/fpsyg.2021.606858

\title{
Neural Responses of Benefiting From the Prosocial Exchange: The Effect of Helping Behavior
}

\begin{abstract}
Daniele Olivo 1,2, Andrea Di Ciano ${ }^{3}$, Jessica Mauro², Lucia Giudetti ${ }^{3}$, Alan Pampallona ${ }^{3}$, Katharina M. Kubera ${ }^{4}$, Dusan Hirjak ${ }^{5}$, Robert Christian Wolf ${ }^{4}$ and Fabio Sambataro ${ }^{1,2,6 *}$
\end{abstract}

${ }^{1}$ Department of Neuroscience (DNS), University of Padua, Padua, Italy, ${ }^{2}$ Department of Medicine (DAME), University of Udine, Udine, Italy, ${ }^{3}$ Fondazione Giancarlo Quarta, Milan, Italy, ${ }^{4}$ Center for Psychosocial Medicine, Department of General Psychiatry, Heidelberg University, Heidelberg, Germany, ${ }^{5}$ Department of Psychiatry and Psychotherapy, Central Institute of Mental Health, Medical Faculty Mannheim, Heidelberg University, Mannheim, Germany, ${ }^{6}$ Padua Neuroscience Center, University of Padua, Padua, Italy

Prosocial behavior is critical for the natural development of an individual as well as for promoting social relationships. Although this complex behavior results from gratuitous acts occurring between an agent and a recipient and a wealth of literature on prosocial behavior has investigated these actions, little is known about the effects on the recipient and the neurobiology underlying them. In this study, we used functional magnetic resonance imaging to identify neural correlates of receiving prosocial behavior in the context of real-world experiences, with different types of action provided by the agent, including practical help and effort appreciation. Practical help was associated with increased activation in a network of regions spanning across bilateral superior temporal sulcus, temporoparietal junction, temporal pole, and medial prefrontal cortex. Effort appreciation was associated with activation and increased task-modulated connectivity of the occipital cortex. Prosocial-dependent brain responses were associated with positive affect. Our results support the role of the theory of mind network and the visual cortices in mediating the positive effects of receiving gratuitous help. Moreover, they indicate that specific types of prosocial behavior are mediated by distinct brain networks, which further demonstrates the uniqueness of the psychological processes underlying prosocial actions.

Keywords: prosocial exchange, practical help, effort appreciation, fMRI, helping behavior, theory of mind

\section{INTRODUCTION}

Prosocial behaviors are a set of acts aimed directly at benefiting people other than oneself, and they entail helping, comforting, cooperation, and kindness (Batson, 2012). This definition comprises a broad number of behaviors ranging from providing support in response to an immediate physical threat to someone else, to helping someone to carry the grocery, or to listening supportively to the other person's predicaments. Although by definition, prosocial behaviors are altruistic in nature and sometimes they may have negative consequences for the person that delivers them, they have positive physical and mental long-term effects (Inagaki and Eisenberger, 2016; 
Wang et al., 2020). Indeed, these behaviors, including volunteering, have been associated with lower levels of depression (Kim and Pai, 2010) and greater mental wellbeing and satisfaction in life (Tabassum et al., 2016). Not only society but also the individual can benefit from carrying out this behavior. It is noteworthy that kind behavior predicted an increase in subjective happiness, which can mediate positive effects on well-being as well as on social relationships, and this can play an adaptive role (Otake and Fredrickson, 2012).

Although the agent, who performs the actions, and the recipient, who benefits from them, have both fundamental roles in prosocial behavior, the literature on the neural effects of this interaction on the recipient is quite limited. Indeed, receiving help can cause mixed moral emotions (Fisher et al., 1982), including gratitude, indebtedness, and guilt, and the type of elicited psychological responses can affect social exchanges and ultimately interpersonal relationships (Algoe, 2012). In particular, gratitude is a positive interpersonal emotion deriving from the combination of the appreciation of the action and the pleasure of enjoying the action itself (McCullough, 2002). The recipient most frequently experiences this emotion when the agent is thought to have benevolent intentions (Tsang, 2006), to give gratuitously (Watkins et al., 2006), and to sustain a cost and that the action that is received is valuable (Tsang, 2006). Indebtedness is more often a negative emotion associated with discomfort, which stems from the perceived need to reciprocate another to restore equity in social interactions. Guilt may also arise from a prosocial interaction. This is a negative emotion associated with violating moral codes, which is associated with indebtedness and reflects the expectation of failing to reciprocate for timing (e.g., too early or too late) or inability to do it properly (Layous et al., 2017). Although traditionally studied as separate entities, these emotions tend to co-exist in social exchanges. Several factors can contribute to the experience of these emotions with respect to a prosocial interaction: traits of personality, such as self-focused attention, can favor indebtedness and particularly in those individuals more prone to perceive themselves as social objects and to have greater negative selfevaluation (Mathews and Green, 2010); cultural determinants play also a role, with individuals from Eastern Asia feeling greater indebtedness relative to Western people (Hitokoto, 2016).

Furthermore, within prosocial behaviors, the type of gratuitous help, although similar for intent and purpose, may differ in the level of autonomy of the receiver (Pierce et al., 1997). For example, in a medical relationship, a patient can let the doctor decide about the treatment or make an informed decision, where the doctor has only a supportive function in the decision. These two polar approaches are practical help and effort appreciation. Practical help is delivered when the agent performs a behavior directly on the source of the problem, and the receiver passively benefits from material aid, dependency-oriented help, e.g., helping in carrying heavy bags or providing a meal. On the other hand, effort appreciation is the conduct whereby the agent values the efforts of the receiver at resolving a specific issue, autonomy-oriented help, e.g., commenting positively on quitting smoking or having a healthier diet. Although both types of aid can be perceived as prosocial and positive, the role of the receiver is different. In the practical help condition, the receiver passively benefits from an immediate gratuitous help/aid, and this is generally associated with gratitude and/or indebtedness (Lane and Anderson, 1976). In the effort appreciation, the receiver actively performs an action/behavior, and a positive feedback strategy is delivered; again, this is associated with positive affect and its effects can foster subsequent learning that is crucial during the development stages of life (Qu and Zelazo, 2007) as well as for promoting changes linked with healthier lifestyle (Young, 2014).

Recent studies have started to identify the brain networks underlying prosocial behaviors (Telzer et al., 2011; Lockwood et al., 2016; Will et al., 2016; Lamm et al., 2019). The medial and lateral parts of the prefrontal cortex and the anterior cingulate have been associated with the computation of the cost-benefit value of an action (Apps and Ramnani, 2014) and its social and moral assessment as well as with mediating the cognitive conflict between personal interests and the other's needs (Cohen and Lieberman, 2010). The medial prefrontal cortex (mPFC), temporoparietal junction (TPJ), and posterior superior temporal sulcus (pSTS) have been implicated in mentalizing and perspective taking (Mitchell et al., 2005; Decety and Lamm, 2007; Behrens et al., 2008). In particular, right TPJ has been implicated in the inference of other's effort during movement that is important in estimating the cost of helping, and together with its role in reorienting attention can contribute to higherlevel social cognition (Mizuguchi et al., 2016). Additionally, the default mode network (DMN), which is supposed to mediate self-referential processes such as autobiographical navigation, daydreaming, or emotional introspection (Raichle et al., 2001), has been implicated in the other-referential thinking and social behavior (Andrews-Hanna, 2012; Mars et al., 2012). Indeed, a very recent study on indebtedness found the engagement of a network encompassing the Theory of Mind (i.e., DMPFC and TPJ) as well as the DMN during an interactive game with different conditions of reciprocity (Gao et al., 2020). Furthermore, different regions of sensory motor and DMN may be recruited in processing comments on the self. Positive social feedback in children during a social evaluation paradigm was associated with increased activation in occipital areas, postcentral, striatum, and cingulate cortex (Achterberg et al., 2016). Subjects receiving positive evaluative feedback on the self during a verbal social approval task investigating personal actions in socio-moral context showed increased activation in pre- and post-central regions, occipito-temporal (mainly, lingual gyrus), and cingulate cortex (van Schie et al., 2018).

In the present study, we used functional magnetic resonance imaging (fMRI) to characterize the neural correlates of receiving prosocial behavior. To have greater ecological validity, to elicit the brain responses associated with this prosocial behavior, we used vignettes portraying practical help and effort appreciation that were depicted in real life situations (Knutson et al., 2010). To avoid the type of help by valence interaction, we limited our study to situations that elicited positive affect. Our first goal was to characterize the neural correlates underlying receiving gratuitous support. Secondly, we wanted to identify the differential mechanisms underlying specific types of aid. 
Third, given the association of positive affect and altruism, we tested whether brain responses underlying received prosocial behavior were associated with positive affect. Based on previous literature, we anticipate that practical help will elicit greater activation of the ToM and DMN networks (Mizuguchi et al., 2016; Gao et al., 2020) for their role in mediating the direct costs of social interaction and indebtedness. On the other hand, effort appreciation, which entails social approval for the self, is expected to be associated with a prefrontal-cingulate network along with brain regions involved in visual perception as suggested by previous studies on social reward (Achterberg et al., 2016; Makowski et al., 2016). In addition, we expect that the brain activations associated with helping conditions will be associated with a greater propensity to positive affect during social interactions.

\section{MATERIALS AND METHODS}

\section{Subjects}

Thirty right-handed Italian native speakers (11 males; age range between 19 and 33 years) with reported normal or correctedto-normal vision participated in the study. Exclusion criteria included (1) any history of substance abuse or dependence (excluding nicotine); (2) head trauma with loss of consciousness; (3) any current medical or neurological disease; (4) any personality disorder; (5) documented intellectual impairment; (6) any contraindications for MRI scan; and (7) excessive head motion during MRI scan.

All research participants gave written informed consent to the study, which was approved by the Institutional Review Board of the University of Parma (UNIPRMR750v1) and conducted in accordance with the principles expressed in the Declaration of Helsinki.

\section{Procedure}

During the functional magnetic resonance imaging (fMRI) acquisition, each participant performed a social interaction task.

\section{Stimuli}

Each trial consisted of two parts: a passive presentation of social interactions and an active affect rating task. At the beginning of each trial, a series of 3 vignettes and 3 sentences in between, depicting a social interaction between two people were presented. In the vignettes, a gray-haired character, the receiver, who the participant was requested to identify with, and another person, the agent, interacted in everyday life scenes. The sentences were short descriptive passages ( $\max 2$ lines) in between vignettes with similar length and complexity across conditions, meant to clarify and link the vignettes to help in their mental representation. Each trial portrayed a social interaction, including helping (practical help and effort appreciation) and non-helping (control) conditions, where the receiver performed an action and interacted with other people. In practical help, the receiver, who faces a task, is helped unconditionally by the agent; on the other hand, in effort appreciation, the receiver willingly performs an action/behavior to pursue his/her goals and in doing so is valued by the agent. The non-helping control condition, similar in visual, and conceptual complexity to the social interaction conditions, included a situation where the receiver accomplished a result without direct communication or behavioral intervention by the agent (for more details see Figure 1). After the social interaction, three smileys in a row displaying happy, neutral, and sad faces were presented, and the subject was asked to rate his/her affect relative to the social interaction by a button press. Visual complexity for vignettes, action content, and word length for sentences did not differ across helping conditions (see Supplementary Materials for details).

\section{Design}

Ninety pseudorandomized trials were presented; 60 nonrepeating helping scenarios depicting effort recognition and practical help conditions (30 each) matched for visual and textual complexity, alternated with 30 non-helping control trials that repeated the scenarios of the helping conditions. Each condition trial consisted of a sequence of three 3500ms sentence slides, alternated with three 2500 -ms vignettes, for a total duration of $18000 \mathrm{~ms}$, and was followed by an affect rating task lasting for $3000 \mathrm{~ms}$ (Figure 1). In between the trials, a crosshair was presented for $1590 \mathrm{~ms}$. Before the scan, participants were trained with a brief mock session, during which they were explicitly instructed to empathize with the receiver in the scene.

\section{Image Acquisition}

All MRI images were acquired on a 3.0 T scanner (GE Discovery MR750, Local Health Unit of Parma). For each subject, structural T1-weighted images were acquired by 3D-MPRAGE sequence with the following parameters: $\mathrm{TR}=9700 \mathrm{~ms}$, $\mathrm{TE}=3.97 \mathrm{~ms}$, FOV $=256 \times 256 \mathrm{~mm} 2$, voxel size $=0.5 \times 0.5 \times 0.9 \mathrm{~mm} 3$ fMRI scan was performed using a gradient-echo-planar imaging (GE-EPI) sequence with the following parameters: $\mathrm{TR}=2000 \mathrm{~ms}$, $\mathrm{TE}=30 \mathrm{~ms}$, flip angle $=90^{\circ}, \mathrm{FOV}=240 \times 240 \mathrm{~mm}^{2}$, voxel size $=3.2 \times 3.2 \times 3.5 \mathrm{~mm}^{3}$. The social interaction task scan comprised 338-volumes per each run.

\section{Imaging Preprocessing}

The preprocessing pipeline was run in $\mathrm{DPABI}^{1}$. The scans were visually inspected by FS and DO to exclude artifacts or lesions. All functional and anatomical images were reoriented to set the origin to the anterior commissure and the horizontal axis parallel to the AC-PC line. Then, the functional images were realigned (a least-squares approach and a six-parameter spatial transformation) to correct for head motion and co-registered to the individual structural scan. The anatomical images were segmented and normalized into the Montreal Neurological Institute standard space using the MNI152 template and the estimated parameters were applied to all functional images that were resampled to a $3 \times 3 \times 3 \mathrm{~mm}^{3}$ voxel size. Finally, images were smoothed using a $6 \mathrm{~mm}$ full width at half maximum Gaussian kernel to increase the signal-to-noise ratio and to compensate for residual anatomical variation across subjects.

\footnotetext{
${ }^{1}$ http://rfmri.org/dpabi
} 
A

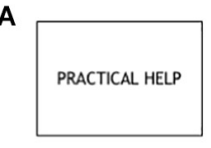

B

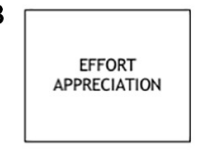

C

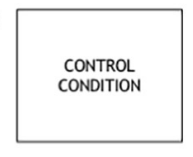

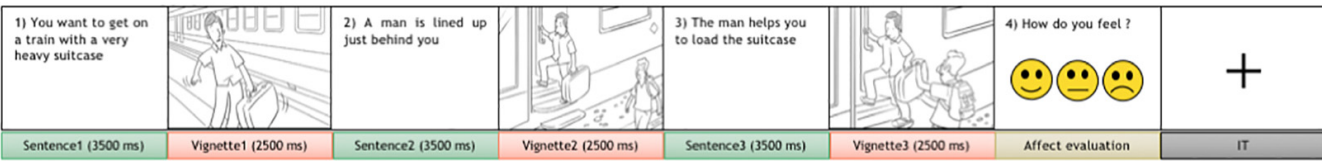

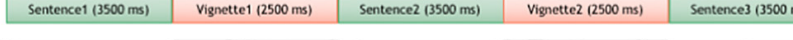

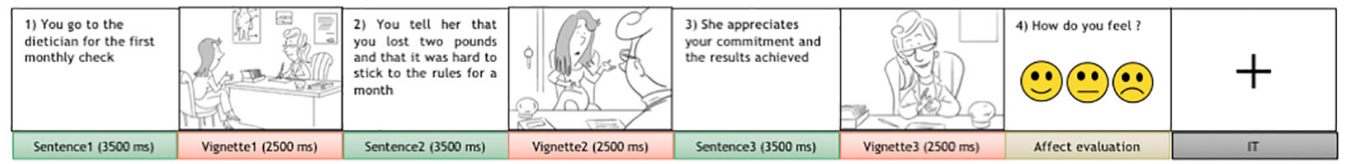

FIGURE 1 | The social interaction task. Each trial included two parts: the display of a social interaction scene composed of three sentences alternated with three vignettes and an affect rating task. A variable duration intertrial interval was presented in between trials. The participant was asked to identify himself/herself with the gray-haired person, the receiver, who interacted with another person, the agent. Three social interaction conditions were presented: two helping conditions, practical help (A), effort appreciation (B), and a non-helping control condition (C).

\section{Imaging Analysis}

The data were analyzed using a general linear model (GLM) with statistical parametrical mapping $\left(\mathrm{SPM} 12^{2}\right)$. The task was modeled with four block regressors: three regressors for the social interaction condition (practical help, effort appreciation, and control) and one for the affect rating task. Each block was modeled with a box-car and convolved with a canonical hemodynamic response function. Six-motion parameters estimated during realignment were included as nuisance covariates. In the first level analyses, whole-brain $\mathrm{t}$-contrast maps of each interacting condition relative to a non-helping control (i.e., practical help greater than control condition and effort appreciation greater than control condition but not the reverse contrast were tested) as well as a direct comparison between social interaction conditions (effort appreciation greater than practical help; practical help greater than effort appreciation) were computed for each subject. In the second-level analyses, individual contrasts were entered in random-effect group analysis (Friston et al., 1999) and onesample $t$-test spatial maps were estimated for each contrast.

Psychophysiological interaction (PPI) analyses were performed to identify task-dependent changes in connectivity between the brain regions identified in the activation analyses. We used the results from group analyses as functional localizer to draw the seeds for the PPI: 5-mm-radius spherical masks centered on the peak coordinates of rTPJ (peak xyz $=63,-9$, 12) (Shamay-Tsoory, 2011; Morelli et al., 2014) for practical help and lingual gyrus (peak xyz $=3,-84,-9$ ) (Fitzgerald et al., 2013; Schiffer et al., 2014) for effort appreciation, respectively, were created. The first eigenvariate of subject time-courses was extracted from these seeds, mean-centered, high-pass filtered, and deconvolved. A general linear model with three regressors was computed for each subject for each PPI using:

\footnotetext{
${ }^{2}$ https://www.fil.ion.ucl.ac.uk/spm/software/spm12/
}

a physiological regressor (the time course from the seed), a psychological regressor (multiple conditions designed in the social interaction task), and a psychophysiological interaction term, calculated as the cross-product of the physiological and psychological regressors. Individual PPI contrasts were entered into random-effects one-sample $t$-tests testing for increased and reduced brain connectivity in the helping relative to the control condition.

A whole-brain family-wise error-corrected threshold of $p<0.05$ at the cluster level with a cluster-defining threshold of uncorrected $p<0.001$ at the voxel-level was applied using Gaussian random field theory in SPM. The minimum cluster size for the activation analyses was 118 voxels in practical help $>$ control, 926 voxels in effort recognition $>$ control, 97 voxels in practical help > control, and 206 voxels in effort recognition $>$ practical help contrasts. For the PPIs, 176 voxels for negative interaction for practical help, 206 and 260 voxels for positive and negative interaction for effort recognition, respectively.

To identify shared functional connectivity networks that are modulated by prosocial conditions, we created conjunction maps of the statistical maps of PPI effects for practical help and effort appreciation relative to controls condition. Statistical maps were thresholded with $p<0.001$ so that the resulting conjoint probability of the conjunction maps was $p<10-6$ (Sambataro et al., 2012).

To infer the mental processes associated with activation and connectivity in specific brain regions from published fMRI studies, a functional decoding approach implemented in Neurosynth database ${ }^{3}$ was used. Briefly, the contrast maps for each prosocial condition and PPI was uploaded to the database and voxel-wise Pearson's correlations with each of 400 topic-based meta-analysis maps were carried out (see

\footnotetext{
${ }^{3}$ Neurosynth.org
} 
Yarkoni et al., 2011 for details on Neurosynth methods) (Yarkoni et al., 2011). The 10 highest correlated items for each map are reported in the Supplementary Materials (Supplementary Tables S1 and S2).

\section{Behavioral Analysis}

The behavioral responses, recorded during the affect evaluation part of the fMRI task for each social interaction condition, were averaged for each participant and analyzed offline. The altruistic nature of the help received in the scenes was expected to elicit positive affective responses. For this reason, affect responses were binarized into positive and negative responses, this latter group including button presses indicating negative and neutral affect. Positive responses showed high negative skewness for helping conditions and positive skewness for non-helping conditions (Supplementary Figure S2A), conversely, negative responses showed high positive skewness for helping conditions and negative skewness for non-helping conditions (Supplementary Figure S2B). The proportion of positive responses across social interaction conditions was compared using chi-square tests. To assess the tendency to respond positively to helping behavior, we used a signal-detection theory approach (Green and Swets, 1966). We indexed performance according to participants' ability to identify the altruistic nature of a scene based on a positive affect response relative to a non-altruistic condition. Positive responses to helping conditions were considered hits, negative/neutral responses to the non-altruistic condition were considered correct rejects, and positive responses to the non-altruistic condition were considered false alarms. We calculated C-score by averaging the $z$-score that corresponds to the hit rate and the $z$-score that corresponds to the false alarm rate (Green and Swets, 1966). This index reflects the subject's response strategy, which is in our case the inner propensity to provide a positive affect response to social interactions. One outlier with performance more than three standard deviations away from the mean positive affect ratings for both prosocial conditions was excluded from these analyses. To test whether the participant's sex could influence the affect ratings we used three-way ANOVAs with participant's sex, task condition, recipient's sex as predictors, and positive affect ratings as the dependent variable.

To control for personality traits, participants completed also the Big Five Questionnaire (BFQ) for the measurement of the Big Five-Factor Model (which includes the factors Extraversion, Agreeableness or Friendliness, Conscientiousness, Emotional Stability or Neuroticism, and Intellect or Openness to Experience) (Caprara et al., 1993).

\section{Brain Behavior Correlations}

The first eigenvariate of the contrast images for each social interaction condition for each subject within an 8 -mm-radius sphere centered on the peak clusters/subclusters was extracted from significant clusters at the group level. For each social interaction condition, brain responses were correlated with the respective $\mathrm{C}$-score, indicating the propensity to provide a positive response as well as with each BFQ dimension. Bonferroni correction was used to correct for multiple comparisons.

\section{RESULTS}

\section{Behavioral Results}

The affect following practical help $(80.5 \pm 14.0 \%$, mean \pm SD) as well as effort appreciation (82.4 $\pm 16.3 \%)$ trials were rated more positively than in control condition $(15.3 \pm 9.5 \%$; see Supplementary Materials for the distribution of response type per condition across subjects) and this difference was significant for both prosocial interaction conditions (practical help $>$ control condition, $\chi^{2}=87.1, p<0.001$; effort appreciation $>$ control condition, $\chi^{2}=89.8, p<0.001$ ), but not between them $(p>0.1)$. The C-scores for prosocial conditions indicated a greater propensity to attribute to both of them (practical help, $0.08 \pm 0.33$; effort appreciation, $0.03 \pm 0.41$ ) a positive valence relative to the control condition. No difference in propensity was present between conditions $(p>0.1)$. We did not find any effect of participant's-responder's sex match/mismatch ( $p>0.1$; see Supplementary Material S.6. for further details).

\section{Social Interaction Task}

\section{Main Effect of Social Interaction Condition}

During practical help, the posterior region of the middle temporal-occipital cortex (rMTOC, $z=5.78, p=3.7^{*} 10^{-9}$ ), posterior middle temporal gyrus (pMTG), left middle occipital cortex (lMOC, $z=6.18, p=3.3^{*} 10^{-10}$ ), right temporal gyrus (rTG, $\left.z=4.71, p=1.3^{*} 10^{-6}\right)$, precuneus $\left(z=4.65, p=1.7^{*} 10^{-6}\right)$, medial postcentral gyrus $\left(z=4.62, p=1.9^{*} 10^{-6}\right)$ and medial prefrontal cortex (mPFC, $z=4.06, p=2.4^{*} 10^{-5}$ ) showed greater activation relative to control condition (see Table 1 and Figures 2A-C). Effort appreciation revealed greater activation of the visual cortex relative to the control condition, particularly the lingual gyrus $\left(z=5.62, p=9.8^{*} 10^{-9}\right.$ ) (see Table 1 and Figure 2D,E).

TABLE 1 | Montreal Neurological Institute coordinates of weighted centers and peaks of clusters from the two main contrasts: practical help > control condition and effort appreciation > control condition.

\begin{tabular}{|c|c|c|c|c|c|}
\hline \multirow[t]{2}{*}{ Cluster location } & \multirow[t]{2}{*}{ z-score } & \multicolumn{3}{|c|}{ Peak coordinates } & \multirow[t]{2}{*}{$K$-size } \\
\hline & & $x$ & $\mathbf{Y}$ & $\mathbf{z}$ & \\
\hline \multicolumn{6}{|l|}{$\begin{array}{l}\text { Practical help > control } \\
\text { condition }\end{array}$} \\
\hline Middle occipital cortex & 6.18 & -45 & -75 & 15 & 1592 \\
\hline $\begin{array}{l}\text { Middle temporal-occipital } \\
\text { cortex }\end{array}$ & 5.78 & 54 & -66 & 9 & 995 \\
\hline $\begin{array}{l}\text { Middle temporal-occipital } \\
\text { cortex }\end{array}$ & 5.41 & 45 & -60 & 12 & 995 \\
\hline Middle temporal gyrus & 4.71 & 63 & -9 & 12 & 118 \\
\hline Precuneus & 4.65 & 3 & -57 & 45 & 314 \\
\hline $\begin{array}{l}\text { Postcentral gyrus medial } \\
\text { segment }\end{array}$ & 4.62 & -9 & -42 & 63 & 182 \\
\hline Medial prefrontal cortex & 4.06 & -6 & 54 & -9 & 193 \\
\hline \multicolumn{6}{|l|}{$\begin{array}{l}\text { Effort } \\
\text { appreciation > control } \\
\text { condition }\end{array}$} \\
\hline Lingual gyrus & 5.62 & 3 & -84 & -9 & 926 \\
\hline
\end{tabular}




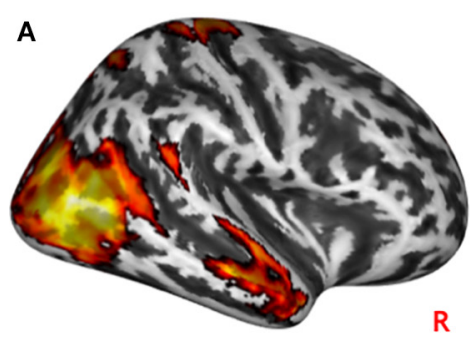

B

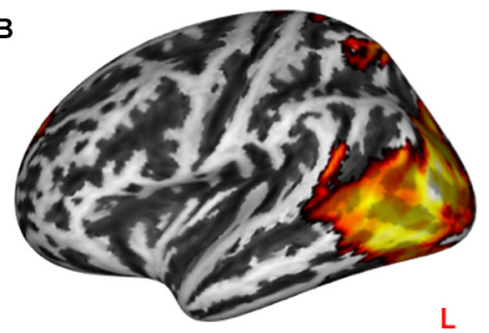

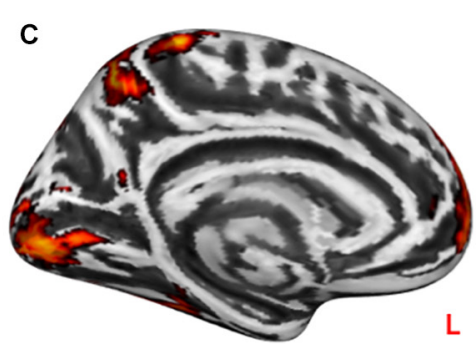
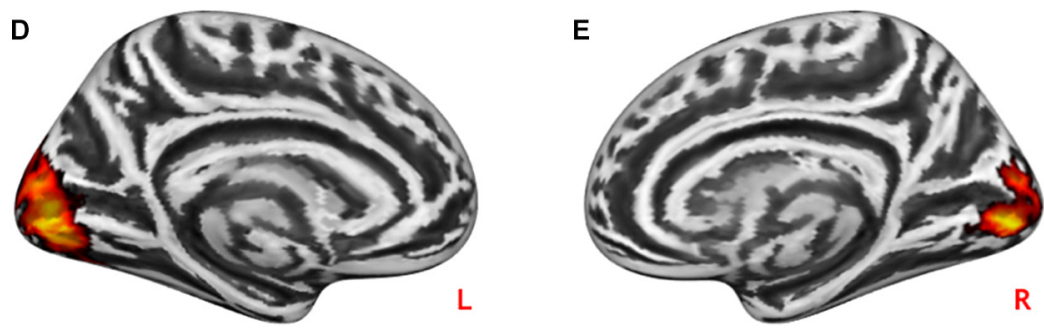

FIGURE 2 | Neural effects of social interactions. Practical help was associated with increased activation in the medial prefrontal as well as temporo-parieto-occipital regions in both the right $\mathbf{( A )}$ and left $(\mathbf{B}, \mathbf{C})$ hemispheres. Effort appreciation was associated with increased left (D) and right (E) occipital activation. Statistical probability maps are rendered on an MNI template with a threshold of voxel-wise $p<0.001$ and FWE-corrected $p<0.05$ at the cluster level. MNI, Montreal Neurological Institute; FWE, family-wise error. L and R indicate left and right brain hemisphere, respectively.

\section{Between Conditions Comparisons}

Practical help showed greater activation in posterior regions of the temporo-occipital cortex (IMTOC, $z=7.03, p=1.0^{*} 10^{-12}$, rMOTC $z=6.94, p=2.0^{*} 10^{-12}$ ) comprising temporoparietal junction (TPJ) and inferior parietal lobule (IPL) but also middle frontal gyrus $\left(z=6.04, p=7.7^{*} 10^{-10}\right)$, superior frontal gyrus $\left(z=5.90, p=1.9^{*} 10^{-9}\right)$ and precuneus $\left(z=7.51, p=2.9^{*} 10^{-14}\right)$ relative to effort appreciation (see Table 2 and Figures 3A-C).

TABLE 2 | Montreal Neurological Institute coordinates of weighted centers and peaks of clusters between the two main contrasts: practical help > effort appreciation and effort appreciation > practical help.

\begin{tabular}{lllll}
\hline Cluster location & Z-score & \multicolumn{2}{r}{ Peak coordinates } & \multirow{2}{*}{ K-size } \\
\cline { 3 - 4 } & & X & Y & Z
\end{tabular}

\section{Practical help > effort}

appreciation

Precuneus

Middle temporal gyrus

Middle occipital gyrus

Fusiform gyrus

Middle frontal gyrus

Superior frontal gyrus

Inferior temporal gyrus

Posterior cingulate gyrus

Effort

appreciation > practical

help

Calcarine gyrus

Lingual gyrus

$\begin{array}{ccccc}7.51 & 9 & -57 & 48 & 1744 \\ 7.03 & -54 & -63 & 0 & 1236 \\ 6.94 & 45 & -66 & 15 & 1528 \\ 6.27 & -30 & -39 & -15 & 118 \\ 6.04 & -24 & 3 & 54 & 713 \\ 5.90 & 24 & 48 & 30 & 1766 \\ 5.10 & 48 & -48 & -21 & 97 \\ 4.60 & -15 & -57 & 6 & 151\end{array}$

1744

1236

1528

118

713

766

151

\section{Brain Behavior Correlations}

Brain activation during practical help was significantly correlated with the $\mathrm{C}$-score for this condition in two clusters within the rMTOC (Supplementary Figure S3): one encompassed the 

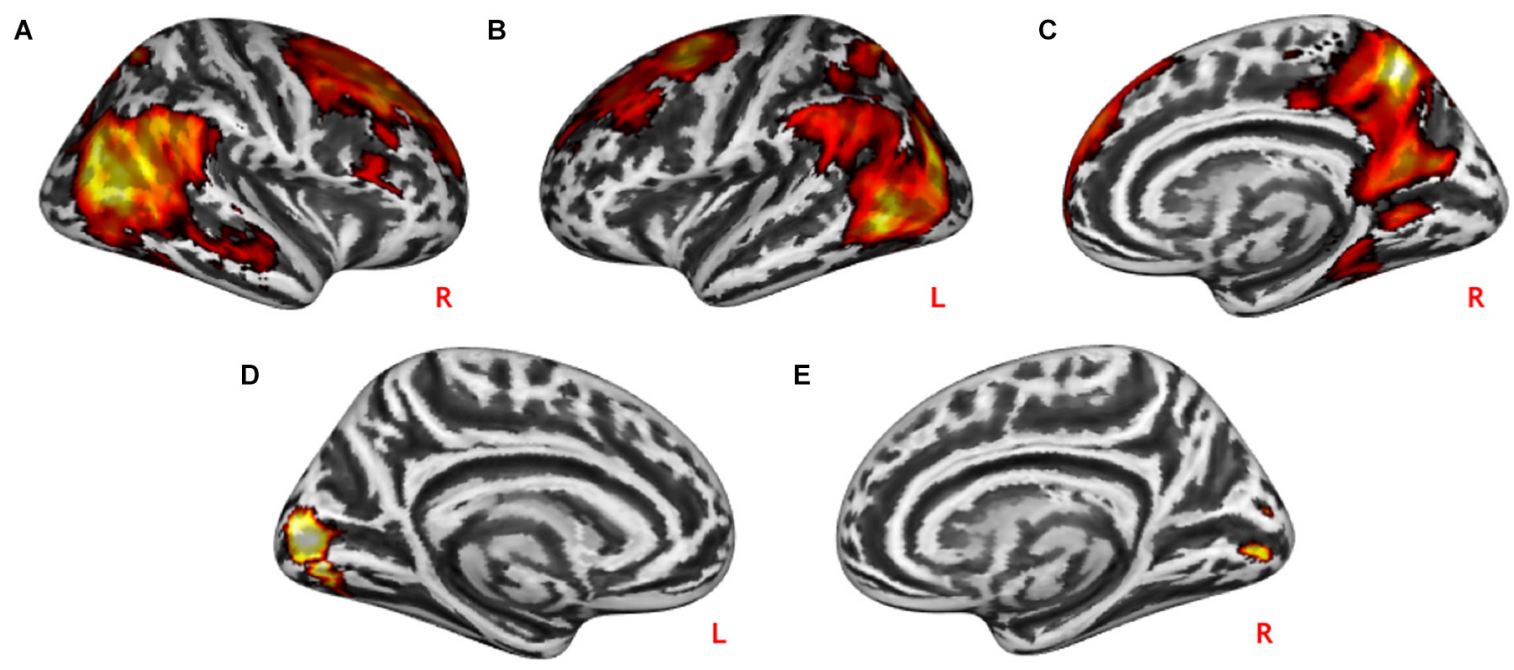

E

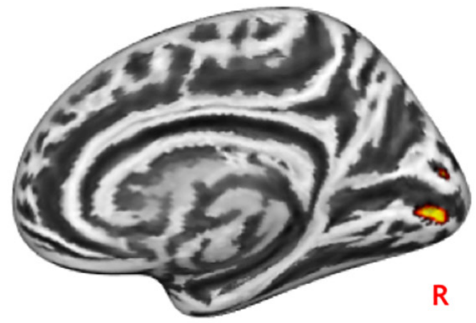

FIGURE 3 | Neural differences between social interaction conditions. Practical help was associated with greater activation in the prefrontal cortex, temporo-parieto-occipital region including TPJ in both the right $(\mathbf{A}, \mathbf{C})$ and left $\mathbf{( B )}$ hemispheres relative to effort appreciation. Effort appreciation was associated with increased left (D) and right (E) occipital activation relative to practical help. Statistical probability maps are rendered on an MNI template with a threshold of voxel-wise $p<0.001$ and FWE-corrected $p<0.05$ at the cluster level. MNI, Montreal Neurological Institute; FWE, family-wise error; TPJ, temporoparietal junction. L and $\mathrm{R}$ indicate left and right brain hemisphere, respectively.

posterior portion of the right middle temporal gyrus and the anterior portion of the right inferior occipital gyrus ( $x y z=54$, $-66,9 ; r=0.42 ; p=0.02$ ), and the second spanned between the right posterior middle temporal gyrus and the angular gyrus $(x y z=45,-60,12 ; r=0.43 ; p=0.02$ ). During effort appreciation, a cluster in the right lingual gyrus showed a significant correlation with the $\mathrm{C}$-score during this condition $($ xyz $=3,-84,-9 ; r=0.43 ; p=0.02)$. We did not find any

TABLE 3 | Montreal Neurological Institute peak coordinates of clusters showing significant psychophysiological interactions for the two task conditions relative to control $(p<0.05$ FWE at the cluster level).

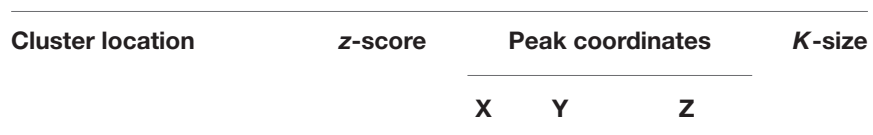

\section{Practical help > control}

\section{condition}

Medial frontal gyrus

Inferior frontal gyrus

Middle temporal gyrus

Middle temporal gyrus

Postcentral gyrus

Inferior parietal lobule

Effort

appreciation > control

\section{condition}

Lingual gyrus

Middle frontal gyrus

Anterior cingulate

$\begin{array}{ccccc}4.42 & 3 & -87 & -9 & 267 \\ -4.69 & 45 & 36 & 27 & 565 \\ -4.04 & 3 & 39 & 24 & 260\end{array}$

correlation between performance and the activation in other clusters that showed an effect of prosocial condition. All the brain behavior correlations did not survive multiple comparison correction using Bonferroni. PPIs did not show any correlation with C-scores for any task condition. We did not find any correlation with any BFQ dimension with the signal of the clusters with significant activations and PPIs.

\section{DISCUSSION}

The present study indicates that receiving unconditional help is associated with the activation of distinct brain regions depending on the type of prosocial aid. During practical help, the medial prefrontal cortex, precuneus as well as bilateral temporo-occipital cortices showed greater activation and reduced task-modulated functional connectivity within a prefrontal-parieto-temporal network. Effort appreciation was associated with the activation of the visual cortex along with increased task-modulated functional connectivity within the visual cortex. Moreover, during effort appreciation, the functional connectivity between the visual and right prefrontal cortex was reduced. Furthermore, the brain activations in right temporo-occipital cortices during practical help, and in the right lingual gyrus during effort appreciation, respectively, were correlated with the propensity to attribute a positive response during social interactions.

Practical help entails direct mentalization of the person who provides help, which includes focusing attention, perspectivetaking and estimating the cost-benefit ratio of a behavior (Schilbach et al., 2008; Mars et al., 2012). Practical help was associated with increased activation of the mentalization network, including TPJ, TP, pSTS, mPFC, and PCC activation. Earlier literature has suggested a role of these regions in

The negative Z-score indicates a reduction of the functional connectivity during the task condition. 

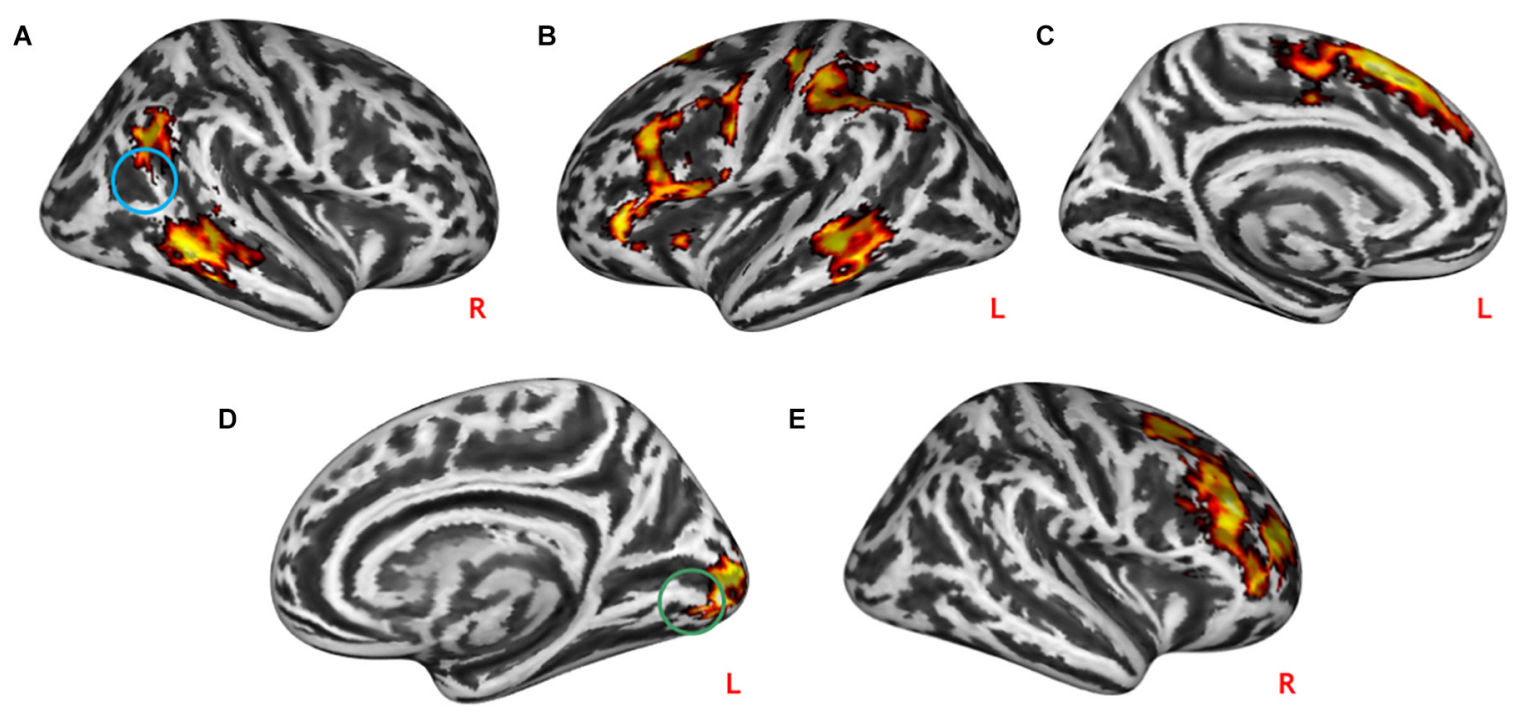

FIGURE 4 | Task-modulated connectivity during social interactions. Practical help was associated with reduced task-modulated connectivity of the right TPJ with the prefrontal cortex, and temporoparietal regions in both the right $\mathbf{( A )}$ and left $\mathbf{( B , C )}$ hemispheres. During effort appreciation, the task-modulated connectivity of the lingual gyrus increased with the occipital cortex (D) and decreased with the right prefrontal cortex (E). Statistical probability maps are rendered on an MNI template with a threshold of voxel-wise $p<0.001$ and FWE-corrected $p<0.05$ at the cluster level. The approximate location of the seeds is represented by colored circles (TPJ, blue; lingual gyrus, green). MNI, Montreal Neurological Institute; FWE, family-wise error; TPJ, temporoparietal junction. L and R indicate left and right brain hemisphere, respectively.

perspective-taking, in attributing the intent of other people's actions (Decety and Lamm, 2007) as well as in the understanding of the kinematics of actions (Saxea and Kanwishera, 2003; Frita and Frith, 2006; Kucyi et al., 2012). In practical help, these regions and pSTS, in particular, can contribute to the estimation of the benefit of an action to one person through

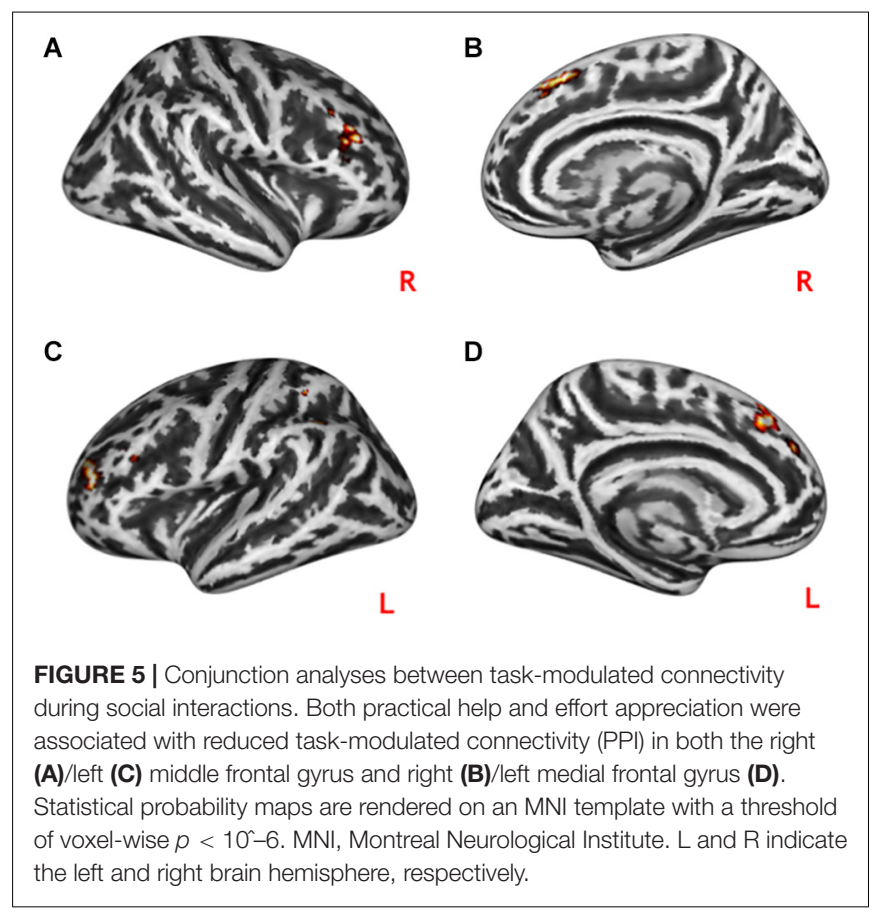

the updating of the expected value representations that are primarily coded by the mPFC (Hampton et al., 2008) as well as via shifting the focus of attention from the self to the others (Mitchell, 2008). The engagement of the entire ToM network is crucial for grasping the effects of receiving practical help and to reason on how the others' mental states can be affected by a behavior (Mars et al., 2012). Similarly, increased activation in $\mathrm{mPFC}$ and greater functional coupling with rTPJ is consistent with the two cognitive roles that this region plays in practical help: social cognitive processing, including mentalization (Castelli et al., 2000; Rameson et al., 2012; Chavez and Heatherton, 2015; Majdandžić et al., 2016), attribution of the self (Schilbach et al., 2008; Mars et al., 2012), and reward processing (Morelli et al., 2015); cognitive processing for the understanding of the social context and in adapting goal-directed behavior to social outcomes (Powers et al., 2015). Additionally, the engagement of parietotemporal regions during practical help can support high-level cognitive and emotional processing that is needed to understand cognitive information associated in social contexts. These findings are in line with previous imaging literature indicating a recruitment of parieto-temporal cortex in cognitive and emotional processes, including focusing attention, memory, language (McKell Carter and Huettel, 2013; Krautheim et al., 2019). In particular, within lateral temporal cortex we found that pMTG activation was associated with the propensity to experience positive affect in vignettes depicting social interactions. This activation has been reported when expectations of social responses are met (Hampton et al., 2008; Hare et al., 2010) and is consistent with the idea that these regions in practical help support not only intention attribution but also contribute to the affective processing associated with 
affiliative behavior that may support prosocial exchanges in the long run (Masten et al., 2011). Overall, findings suggest that the posterior regions of the ToM network during practical help may contribute to the perception of "feeling understood" and predict the proneness to have a greater closeness with the actor in a social interaction, which is partly mediated by the mPFC (Morelli et al., 2013).

Effort appreciation was associated with increased activation along with increased functional coupling of the occipital cortex. The lack of activation of prefrontal-cortical-striatal regions during effort appreciation may be due to a high-level control condition that activated similarly to these regions. Our study suggests that the visual cortex is a central node for effort appreciation processing and its over-recruitment may be linked with reward processing as indicated by the association between its engagement and the propensity to experience positive affect in vignettes containing social interactions. The role of visual cortex in reward processing during this condition may ultimately drive social learning. Although earlier studies did not provide evidence of the role of the visual cortex in social reward processing, recent fMRI literature has shown that sensory cortices, including the visual cortex, can significantly contribute to its representation (Brosch et al., 2011; Weil et al., 2015), and can promote social evaluation and reward (Achterberg et al., 2016; Makowski et al., 2016). Notably, the representation of rewarded stimuli can provide traces that are fundamental for a future learning process (Fitzgerald et al., 2013; Schiffer et al., 2014). Increased engagement of the visual cortex could be driven by top-down mechanisms led of attentional modulation by reward processing, including social reward, and learning-dependent signals (Kim and Anderson, 2020). In other words, in the context of social interaction, the acknowledgment of the subject's efforts by other people may facilitate the performance of the efforts themselves, and therefore the probability of repetition, via the enhancement of learning processes (Kim and Anderson, 2020).

At last, we found reduced connectivity of the rTPJ and lingual gyrus with the prefrontal cortex is reduced during both helping behavior conditions. Prefrontal networks are responsible for executive functions and motor planning. Although relevant for social cognitive processes (Wade et al., 2018), these functions may be deactivated during mentalization to support selfreferential processing, taking the perspective of others and social evaluations, which are needed in both task conditions (Andrews-Hanna, 2012).

Overall, we found greater recruitment of occipital regions during effort appreciation relative to practical help. Several factors including arousal levels and reward processes could have contributed to these findings. Emotional scenes with high arousal may increase activation of the visual cortex via amygdalar efferents (Sabatinelli et al., 2011) as well as via attentional modulation (Murray and Wojciulik, 2004) that in its turn may depend on the relevance of the scene (Niu et al., 2012). Although these processes may be influenced individually by personality traits, we did not find such a relationship in our study. Additionally, the social reward processing component of the scenes could also affect brain activation in the visual cortex (see above) but is not associated with mentalization (Alkire et al., 2018). On the other hand, practical help involves greater recruitment of the theory of mind network for its mentalizing component, whereby the arousal and rewarding component associated with these scenes may be minimal. Notably, both effort appreciation and practical help are associated with reduced connectivity with the right dorsolateral prefrontal regions during benefiting of prosocial behavior, which may underlie a shared process of reallocating resources to socially-laden interactions rather than cognitive processing (see above). Future studies addressing the relevance of arousal, emotional, and reward processing are warranted.

From a clinical point of view, the results of this study may also contribute to a better understanding of aberrant prosocial behavior and social interaction in autism and schizophrenia (Sasson et al., 2011; Fernandes et al., 2018). Modulation of these brain regions in the context of disorderspecific psychotherapeutic interventions and improved prosocial behavior in patients with autism and schizophrenia could lead to an increase in social functioning and quality of life (Vass et al., 2018; Holopainen et al., 2019). Finally, the function of the identified regions may be used as potential biomarkers in patients with autism and schizophrenia (and in other psychiatric disorders with disturbances of social interaction) in clinical studies.

We acknowledge some limitations of this study. First, our results rely on real-world interactions that were modeled using block design, which have greater ecological power but the limited ability to identify the brain activity associated with each cognitive process implicated during the social interaction. Second, in our study, to reduce the heterogeneity and increase the power to detect a significant effect, we investigated only a limited type of prosocial interactions. Lastly, the sample size of our study may have not been able to detect all the effects associated with prosocial interactions. Nonetheless, this sample size falls in the average range of participants for similar studies in the moral judgment (Garrigan et al., 2016) and theory of mind (Schurz et al., 2014) field. Future studies investigating other types of prosocial interactions and their subprocesses are warranted.

\section{CONCLUSION}

Taken together, the current findings highlight the pivotal role of the brain regions supporting ToM as well as the visual cortex during receiving prosocial behavior. Overall, our findings suggest that in social situations, the brain activity in these networks reflects not only the match between the individual's needs and the other's actions but may also promote the social interaction directly and through learning via positive affect. From a clinical point of view, progress in this research area can contribute to a better understanding of the physician-patient relationship, where the individual can express needs and show efforts of change that prompt the physician to respond. Furthermore, our results can inform the literature on neurodevelopmental disorders, such 
as autism and schizophrenia, in which social interactions are crucially impaired (Fernandes et al., 2018).

\section{DATA AVAILABILITY STATEMENT}

The datasets presented in this study can be found in online repositories. The names of the repository/repositories and accession number(s) can be found below: https://neurovault.org/ collections/9321/, https://osf.io/2gt8m.

\section{ETHICS STATEMENT}

The studies involving human participants were reviewed and approved by the Comitato Etico per Parma. The patients/participants provided their written informed consent to participate in this study.

\section{AUTHOR CONTRIBUTIONS}

FS, AP, AD, and LG conceived of the experiments. DO and JM performed the experiments and data analyses. FS and DO wrote an initial version of the manuscript. All authors contributed to the interpretation of the results, provided comments, and approved of the final version.

\section{REFERENCES}

Achterberg, M., van Duijvenvoorde, A. C. K., Bakermans-Kranenburg, M. J., and Crone, E. A. (2016). Control your anger! The neural basis of aggression regulation in response to negative social feedback. Soc. Cogn. Affect. Neurosci. 11, 712-720. doi: 10.1093/scan/nsv154

Algoe, S. B. (2012). Find, remind, and bind: the functions of gratitude in everyday relationships. Soc. Personal. Psychol. Compass 6, 455-469. doi: 10.1111/j.17519004.2012.00439.x

Alkire, D., Levitas, D., Warnell, K. R., and Redcay, E. (2018). Social interaction recruits mentalizing and reward systems in middle childhood. Hum. Brain Mapp. 39, 3928-3942. doi: 10.1002/hbm.24221

Andrews-Hanna, J. R. (2012). The brain's default network and its adaptive role in internal mentation. Neuroscientist 18, 251-270. doi: 10.1177/ 1073858411403316

Apps, M. A. J., and Ramnani, N. (2014). The anterior cingulate gyrus signals the net value of others' rewards. J. Neurosci. 34, 6190-6200. doi: 10.1523/jneurosci. 2701-13.2014

Batson, C. D. (2012). “A history of prosocial behavior research," in Handbook of the History of Social Psychology, eds A. W. Kruglanski and W. Stroebe (New York, NY: Psychology Press), doi: 10.4324/9780203808498.ch12

Behrens, T. E. J., Hunt, L. T., Woolrich, M. W., and Rushworth, M. F. S. (2008). Associative learning of social value. Nature 456, 245-250. doi: 10.1038/ nature 07538

Brosch, M., Selezneva, E., Scheich, H., Lewis, J. W., and Virginia, W. (2011). Representation of reward feedback in primate auditory cortex. Front. Syst. Neurosci. 5:5. doi: 10.3389/fnsys.2011.00005

Caprara, G. V., Barbaranelli, C., Borgogni, L., and Perugini, M. (1993). The 'big five questionnaire': a new questionnaire to assess the five factor model. Pers. Individ. Dif. 15, 281-288. doi: 10.1016/0191-8869(93)90218-r

Castelli, F., Happé, F., Frith, U., and Frith, C. (2000). Movement and mind: a functional imaging study of perception and interpretation of complex intentional movement patterns. Neuroimage 12, 314-325. doi: 10.1006/nimg. 2000.0612

\section{FUNDING}

This work was generously supported by an award from the Fondazione Giancarlo Quarta Onlus "Stili relazionali a confronto: analisi delle attivazioni cerebrali connesse a differenti modalità communicative" to FS and by the Italian Ministry of Education, University and Research (MIUR) (program PRIN 2017 grant number 2017K2NEF4 to FS and Department of Excellence 2018-2022 awarded to the Department of Neuroscience-University of Padua).

\section{ACKNOWLEDGMENTS}

FS's research is supported by the Italian Ministry of Education, University and Research (MIUR), programme PRIN 2017 grant number 2017K2NEF4, and Department of excellence 2018-2022 to the Department of Neuroscience - University of Padua. We thank Elena Moro, MD, for helpful discussions on the project.

\section{SUPPLEMENTARY MATERIAL}

The Supplementary Material for this article can be found online at: https://www.frontiersin.org/articles/10.3389/fpsyg. 2021.606858/full\#supplementary-material

Chavez, R. S., and Heatherton, T. F. (2015). Representational similarity of social and valence information in the medial pFC. J. Cogn. Neurosci. 27, 73-82. doi: 10.1162/jocn_a_00697

Cohen, J. R., and Lieberman, M. D. (2010). "The common neural basis of exerting self control in multiple domains," in Self Control in Society, Mind, and Brain, eds Y. Trope, R. Hassin, and K. N. Ochsner (Oxford: Oxford University Press).

Decety, J., and Lamm, C. (2007). The role of the right temporoparietal junction in social interaction: how low-level computational processes contribute to meta-cognition. Neuroscientist 13, 580-593. doi: 10.1177/10738584073046 54

Fernandes, J. M., Cajão, R., Lopes, R., Jerónimo, R., and Barahona-Corrêa, J. B. (2018). Social cognition in schizophrenia and autism spectrum disorders: a systematic review and meta-analysis of direct comparisons. Front. Psychiatry 9:504. doi: 10.3389/fpsyt.2018.00504

Fisher, J. D., Nadler, A., and Whitcher-Alagna, S. (1982). Recipient reactions to aid. Psychol. Bull. 91, 27-54. doi: 10.1037/0033-2909.91.1.27

Fitzgerald, T. H. B., Friston, K. J., and Dolan, R. J. (2013). Characterising reward outcome signals in sensory cortex. Neuroimage 83, 329-334. doi: 10.1016/j. neuroimage.2013.06.061

Friston, K. J., Holmes, A. P., Price, C. J., Büchel, C., and Worsley, K. J. (1999). Multisubject fMRI studies and conjunction analyses. Neuroimage 10, 385-396. doi: 10.1006/nimg.1999.0484

Frita, C. D., and Frith, U. (2006). How we predict what other people are going to do. Brain Res. 1079, 36-46. doi: 10.1016/j.brainres.2005. 12.126

Gao, X., Jolly, E., Yu, H., Liu, H., Zhou, X., Chang, L. J., et al. (2020). The hidden cost of receiving favors: a theory of indebtedness. bioRxiv[Preprint] doi: 10.1101/2020.02.03.926295

Garrigan, B., Adlam, A. L. R., and Langdon, P. E. (2016). The neural correlates of moral decision-making: a systematic review and meta-analysis of moral evaluations and response decision judgements. Brain Cogn. 108, 88-97. doi: 10.1016/j.bandc.2016.07.007

Green, D., and Swets, J. (1966). Signal Detection Theory and Psychophysics. New York, NY: Wiley. 
Hampton, A. N., Bossaerts, P., and Doherty, J. P. O. (2008). Neural correlates of mentalizing-related computations during strategic interactions in humans. Proc. Natl. Acad. Sci. U.S.A. 105, 6741-6746. doi: 10.1073/pnas.0711099105

Hare, T. A., Camerer, C. F., Knoepfle, D. T., O’Doherty, J. P., and Rangel, A. (2010). Value computations in ventral medial prefrontal cortex during charitable decision making incorporate input from regions involved in social cognition. J. Neurosci. 30, 583-590. doi: 10.1523/jneurosci.4089-09.2010

Hitokoto, H. (2016). Indebtedness in cultural context: the role of culture in the felt obligation to reciprocate. Asian. J. Soc. Psychol. 19, 16-25. doi: 10.1111/ajsp. 12122

Holopainen, A., de Veld, D. M. J., Hoddenbach, E., and Begeer, S. (2019). Does theory of mind training enhance empathy in Autism? J. Autism Dev. Disord. 49, 3965-3972. doi: 10.1007/s10803-018-3671-1

Inagaki, T. K., and Eisenberger, N. I. (2016). Giving support to others reduces sympathetic nervous system-related responses to stress. Psychophysiology 53, 427-435. doi: 10.1111/psyp. 12578

Kim, A. J., and Anderson, B. A. (2020). Neural correlates of attentional capture by stimuli previously associated with social reward. Cogn. Neurosci. 11, 5-15. doi: $10.1080 / 17588928.2019 .1585338$

Kim, J., and Pai, M. (2010). Volunteering and trajectories of depression. J. Aging Health 22, 84-105. doi: 10.1177/0898264309351310

Knutson, K. M., Krueger, F., Koenigs, M., Hawley, A., Escobedo, J. R., Vasudeva, V., et al. (2010). Behavioral norms for condensed moral vignettes. Soc. Cogn. Affect. Neurosci. 5, 378-384. doi: 10.1093/scan/nsq005

Krautheim, J. T., Dannlowski, U., Steines, M., Neziroğlu, G., Acosta, H., Sommer, J., et al. (2019). Intergroup empathy: enhanced neural resonance for ingroup facial emotion in a shared neural production-perception network. Neuroimage 194, 182-190. doi: 10.1016/j.neuroimage.2019.03.048

Kucyi, A., Hodaie, M., and Davis, K. D. (2012). Lateralization in intrinsic functional connectivity of the temporoparietal junction with salience- and attentionrelated brain networks. J. Neurophysiol. 108, 3382-3392. doi: 10.1152/jn.00674. 2012

Lamm, C., Rütgen, M., and Wagner, I. C. (2019). Imaging empathy and prosocial emotions. Neurosci. Lett. 693, 49-53. doi: 10.1016/j.neulet.2017.06.054

Lane, J., and Anderson, N. H. (1976). Integration of intention and outcome in moral judgment. Mem. Cognit. 4, 1-5. doi: 10.3758/bf03213247

Layous, K., Sweeny, K., Armenta, C., Na, S., Choi, I., Lyubomirsky, S., et al. (2017). The proximal experience of gratitude. PLoS One 12:e0179123. doi: 10.1371/ journal.pone. 0179123

Lockwood, P. L., Apps, M. A. J., Valton, V., Viding, E., and Roiser, J. P. (2016). Neurocomputational mechanisms of prosocial learning and links to empathy. Proc. Natl. Acad. Sci.U.S.A. 113, 9763-9768. doi: 10.1073/pnas.16031 98113

Majdandžić, J., Amashaufer, S., Hummer, A., Windischberger, C., and Lamm, C. (2016). The selfless mind: how prefrontal involvement in mentalizing with similar and dissimilar others shapes empathy and prosocial behavior. Cognition 157, 24-38. doi: 10.1016/j.cognition.2016.08.003

Makowski, C. S., Lepage, M., and Harvey, P. O. (2016). Functional neural correlates of social approval in schizophrenia. Soc. Cogn. Affect. Neurosci. 11, 445-457. doi: $10.1093 /$ scan $/$ nsv125

Mars, R. B., Neubert, F. X., Noonan, M. P., Sallet, J., Toni, I., Rushworth, M. F. S., et al. (2012). On the relationship between the "default mode network" and the "social brain". Front. Hum. Neurosci. 6:189. doi: 10.3389/fnhum.2012.00189

Masten, C. L., Morelli, S. A., and Eisenberger, N. I. (2011). An fMRI investigation of empathy for 'social pain' and subsequent prosocial behavior. Neuroimage 55, 381-388. doi: 10.1016/j.neuroimage.2010.11.060

Mathews, M. A., and Green, J. D. (2010). Looking at me, appreciating you: selffocused attention distinguishes between gratitude and indebtedness. Cogn. Emot. 24, 710-718. doi: 10.1080/02699930802650796

McCullough, M. E. (2002). Savoring life, past and present: explaining what hope and gratitude share in common. Psychol. Inq. 13, 302-304.

McKell Carter, R., and Huettel, S. A. (2013). A nexus model of the temporal-parietal junction. Trends Cogn. Sci. 17, 328-336. doi: 10.1016/j.tics.2013.05.007

Mitchell, J. P. (2008). Activity in right temporo-parietal junction is not selective for theory-of-mind. Cereb. Cortex 18, 262-271. doi: 10.1093/cercor/bhm051

Mitchell, J. P., Banaji, M. R., and Macrae, C. N. (2005). The link between social cognition and self-referential thought in the medial prefrontal cortex. J. Cogn. Neurosci. 17, 1306-1315. doi: 10.1162/0898929055002418
Mizuguchi, N., Nakata, H., and Kanosue, K. (2016). The right temporoparietal junction encodes efforts of others during action observation. Sci. Rep. 6, 1-8. doi: 10.1038/srep30274

Morelli, S. A., Rameson, L. T., and Lieberman, M. D. (2014). The neural components of empathy: predicting daily prosocial behavior. Soc. Cogn. Affect. Neurosci. 9, 39-47. doi: 10.1093/scan/nss088

Morelli, S. A., Sacchet, M. D., and Zaki, J. (2015). Common and distinct neural correlates of personal and vicarious reward: a quantitative meta-analysis. Neuroimage 112, 244-253. doi: 10.1016/j.neuroimage.2014.12.056

Morelli, S. A., Torre, J. B., and Eisenberger, N. I. (2013). The neural bases of feeling understood and not understood. Soc. Cogn. Affect. Neurosci. 9, 1890-1896. doi: 10.1093/scan/nst191

Murray, S. O., and Wojciulik, E. (2004). Attention increases neural selectivity in the human lateral occipital complex. Nat. Neurosci. 7, 70-74. doi: 10.1038/nn1 161

Niu, Y., Todd, R. M., and Anderson, A. K. (2012). Affective salience can reverse the effects of stimulus-driven salience on eye movements in complex scenes. Front. Psychol. 3:336. doi: 10.3389/fpsyg.2012.00336

Otake, K., and Fredrickson, B. L. (2012). Happy people become happier through kindness: a counting kindnesses intervention Keiko. Changes 29, 997-1003.

Pierce, G. R., Lakey, B., Sarason, I. G., and Sarason, R. B. (eds) (1997). "Autonomous and dependent help-seeking: personality characteristics and the seeking of help," in Sourcebook of Social Support and Personality (Philadelphia, NY: Springer US). doi: 10.1007/978-1-4899-1843-7

Powers, K. E., Chavez, R. S., and Heatherton, T. F. (2015). Individual differences in response of dorsomedial prefrontal cortex predict daily social behavior. Soc. Cogn. Affect. Neurosci. 11, 121-126. doi: 10.1093/scan/nsv096

Qu, L., and Zelazo, P. D. (2007). The facilitative effect of positive stimuli on 3-year-olds' flexible rule use. Cogn. Dev. 22, 456-473.

Raichle, M. E., MacLeod, A. M., Snyder, A. Z., Powers, W. J., Gusnard, D. A., Shulman, G. L., et al. (2001). A default mode of brain function. Proc. Natl. Acade. Sci. U.S.A. 98, 676-682.

Rameson, L. T., Morelli, S. A., and Lieberman, M. D. (2012). The neural correlates of empathy: experience, automaticity, and prosocial behavior. J. Cogn. Neurosci. 24, 235-245. doi: 10.1162/jocn_a_00130

Sabatinelli, D., Fortune, E. E., Li, Q., Siddiqui, A., Krafft, C., Oliver, W. T., et al. (2011). Emotional perception: meta-analyses of face and natural scene processing. Neuroimage 54, 2524-2533.

Sambataro, F., Safrin, M., Lemaitre, H. S., Steele, S. U., Das, S. B., Callicott, J. H., et al. (2012). Normal aging modulates prefrontoparietal networks underlying multiple memory processes. Eur. J. Neurosci. 36, 3559-3567.

Sasson, N. J., Pinkham, A. E., Carpenter, K. L. H., and Belger, A. (2011). The benefit of directly comparing autism and schizophrenia for revealing mechanisms of social cognitive impairment. J. Neurodev. Disord. 4, 87-100. doi: 10.1007/ s11689-010-9068-x

Saxea, R., and Kanwishera, N. (2003). People thinking about thinking people the role of the temporo-parietal junction in "theory of mind". Neuroimage 19, 1835-1842. doi: 10.1016/S1053-8119(03)00230-1

Schiffer, A.-M., Muller, T., Yeung, N., and Waszak, F. (2014). Reward activates stimulus-specific and task-dependent representations in visual association cortices. J. Neurosci. 34, 15610-15620. doi: 10.1523/JNEUROSCI.164014.2014

Schilbach, L., Eickhoff, S. B., Rotarska-Jagiela, A., Fink, G. R., and Vogeley, K. (2008). Minds at rest? Social cognition as the default mode of cognizing and its putative relationship to the 'default system' of the brain. Conscious. Cogn. 17, 457-467. doi: 10.1016/j.concog.2008.03.013

Schurz, M., Radua, J., Aichhorn, M., Richlan, F., and Perner, J. (2014). Fractionating theory of mind: a meta-analysis of functional brain imaging studies. Neurosci. Biobehav. Rev. 42, 9-34. doi: 10.1016/j.neubiorev.2014.01.009

Shamay-Tsoory, S. G. (2011). The neural bases for empathy. Neuroscientist 17, 18-24. doi: 10.1177/1073858410379268

Tabassum, F., Mohan, J., and Smith, P. (2016). Association of volunteering with mental well-being: a lifecourse analysis of a national population-based longitudinal study in the UK. BMJ Open 6, 1-8. doi: 10.1136/bmjopen-2016011327

Telzer, E. H., Masten, C. L., Berkman, E. T., Lieberman, M. D., and Fuligni, A. J. (2011). Neural regions associated with self control and mentalizing are recruited 
during prosocial behaviors towards the family. Neuroimage 58, 242-249. doi: 10.1016/j.neuroimage.2011.06.013

Tsang, J. A. (2006). The effects of helper intention on gratitude and indebtedness. Motiv. Emot. 30, 198-204. doi: 10.1007/s11031-006-9031-z

van Schie, C. C., Chiu, C. D., Rombouts, S. A. R. B., Heiser, W. J., and Elzinga, B. M. (2018). When compliments do not hit but critiques do: an fMRI study into self-esteem and self-knowledge in processing social feedback. Soc. Cogn. Affect. Neurosci. 13, 404-417. doi: 10.1093/scan/nsy014

Vass, E., Fekete, Z., Simon, V., and Simon, L. (2018). Interventions for the treatment of theory of mind deficits in schizophrenia: systematic literature review. Psychiatry Res. 267, 37-47. doi: 10.1016/j.psychres.2018.05.001

Wade, M., Prime, H., Jenkins, J. M., Yeates, K. O., Williams, T., Lee, K., et al. (2018). On the relation between theory of mind and executive functioning: a developmental cognitive neuroscience perspective. Psychon. Bull. Rev. 25, 2119-2140. doi: 10.3758/s13423-018-1459-0

Wang, Y., Ge, J., Zhang, H., Wang, H., and Xie, X. (2020). Altruistic behaviors relieve physical pain. Proc. Natl. Acad. Sci. U.S.A. 117, 950-958. doi: 10.1073/ pnas. 1911861117

Watkins, P. C., Scheer, J., Ovnicek, M., and Kolts, R. (2006). The debt of gratitude: dissociating gratitude and indebtedness. Cogn. Emot. 20, 217-241. doi: 10.1080/ 02699930500172291

Weil, R. S., Dolan, R. J., Furl, N., Ruff, C. C., Symmonds, M., Flandin, G., et al. (2015). Rewarding feedback after correct visual discriminations has both general and specific influences on visual cortex. J. Neurophysiol. 104, 1746-1757. doi: $10.1152 /$ jn.00870.2009

Will, G. J., Crone, E. A., Van Lier, P. A. C., and Güroğlu, B. (2016). Neural correlates of retaliatory and prosocial reactions to social exclusion: associations with chronic peer rejection. Dev. Cogn. Neurosci. 19, 288-297. doi: 10.1016/j. dcn.2016.05.004

Yarkoni, T., Poldrack, R. A., Nichols, T. E., Van Essen, D. C., and Wager, T. D. (2011). Large-scale automated synthesis of human functional neuroimaging data. Nat. Methods 8, 665-670. doi: 10.1038/nmeth.1635

Young, S. (2014). Healthy behavior change in practical settings. Perm. J. 18, 89-92.

Conflict of Interest: The authors declare that the research was conducted in the absence of any commercial or financial relationships that could be construed as a potential conflict of interest.

Copyright (C) 2021 Olivo, Di Ciano, Mauro, Giudetti, Pampallona, Kubera, Hirjak, Wolf and Sambataro. This is an open-access article distributed under the terms of the Creative Commons Attribution License (CC BY). The use, distribution or reproduction in other forums is permitted, provided the original author(s) and the copyright owner(s) are credited and that the original publication in this journal is cited, in accordance with accepted academic practice. No use, distribution or reproduction is permitted which does not comply with these terms. 